\title{
Dissociating Visual and Motor Directional Selectivity Using Visuomotor Adaptation
}

\author{
(DShlomi Haar, ${ }^{1,4}$ Opher Donchin, ${ }^{2,4}$ and Ilan Dinstein ${ }^{1,3,4}$ \\ Departments of ${ }^{1}$ Brain and Cognitive Sciences, ${ }^{2}$ Biomedical Engineering, and ${ }^{3}$ Psychology, and ${ }^{4}$ Zlotowski Center for Neuroscience, Ben-Gurion University \\ of the Negev, Beer-Sheva 8410501, Israel
}

\begin{abstract}
Directional selectivity during visually guided hand movements is a fundamental characteristic of neural populations in multiple motor areas of the primate brain. In the current study, we assessed how directional selectivity changes when reaching movements are dissociated from their visual feedback by rotating the visual field. We recorded simultaneous movement kinematics and fMRI activity while human subjects performed out-and-back movements to four peripheral targets before and after adaptation to a $45^{\circ}$ visuomotor rotation. A classification algorithm was trained to identify movement direction according to voxel-by-voxel fMRI patterns in each of several brain areas. The direction of movements was successfully decoded with above-chance accuracy in multiple motor and visual areas when training and testing the classifier on trials within each condition, thereby demonstrating the existence of directionally selective fMRI patterns within each stage of the experiment. Most importantly, when training the classifier on baseline trials and decoding rotated trials, motor brain areas exhibited above-chance decoding according to the original movement direction and visual brain areas exhibited above-chance decoding according to the rotated visual target location, while posterior parietal cortex (PPC) exhibited chance-level decoding according to both. These results reveal that directionally selective fMRI patterns in motor system areas faithfully represent movement direction regardless of visual feedback, while fMRI patterns in visual system areas faithfully represent target location regardless of movement direction. Directionally selective fMRI patterns in PPC, however, were altered following adaptation learning, thereby suggesting that the novel visuomotor mapping, which was learned during visuomotor adaptation, is stored in PPC.
\end{abstract}

Key words: directional selectivity; fMRI; MVPA; out-and-back reaching; visuomotor rotation

\section{Introduction}

Neural populations in many motor areas, including primary motor cortex (M1; Georgopoulos et al., 1982), posterior parietal cortex (PPC; Kalaska et al., 1983), dorsal premotor cortex (PMd; Fu et al., 1993), supplementary motor area (SMA; Lee and Quessy, 2003), and cerebellum (Fortier et al., 1989), exhibit directional tuning during visually guided hand movements. In most previous studies, subjects observed the target location and hand movement such that directional selectivity may have been driven by either the visual information regarding the target location or by the motor commands generating the movement. Dissociating the visual and motor components of directional tuning is possible through the use of visuomotor rotation tasks, which introduce a constant angular difference between the movement of the hand and the location of the target (as well as the direction of the cursor).

\footnotetext{
Received Jan. 14, 2015; revised March 19, 2015; accepted March 21, 2015.

Author contributions: S.H., O.D., and I.D. designed research; S.H. performed research; S.H. analyzed data; S.H., O.D., and I.D. wrote the paper.

This research was supported by grants from the Israel Science Foundation and the German-Israeli Foundation for Scientific Research (I.D.), the Helmsley Foundation (0.D.), and the Agricultural, Biological, and Cognitive Robotics Initiative of Ben-Gurion University of the Negev. We thank Ilan Shelef and Moti Salti for their help in acquiring the fMRI data, and Lior Shmuelof for helpful discussions about the manuscript.

The authors declare no competing financial interests.

Correspondence should be addressed to Shlomi Haar, Ben-Gurion University of the Negev, P.0.B. 653 Beer-Sheva 8410501, Israel. E-mail: haar@post.bgu.ac.il.

DOI:10.1523/JNEUROSCI.0182-15.2015

Copyright $\odot 2015$ the authors $\quad 0270-6474 / 15 / 356813-09 \$ 15.00 / 0$
}

Assessing the selectivity of motor neural populations in humans is possible through the use of multivariate pattern analysis (MVPA) techniques applied to voxel-by-voxel fMRI responses (Dinstein et al., 2008; Gallivan et al., 2011; Kadmon Harpaz et al., 2014; Wiestler et al., 2014). Several recent fMRI studies have reported that directionally selective responses are apparent in human M1 (Cowper-Smith et al., 2010; Eisenberg et al., 2010; Fabbri et al., 2010; Toxopeus et al., 2011), PMd (Cowper-Smith et al., 2010; Fabbri et al., 2010), SMA (Cowper-Smith et al., 2010), and PPC (Fabbri et al., 2010). Of these, one study (Eisenberg et al., 2011) examined directional selectivity before and after visuomotor adaptation in M1 during the execution of joystick movements. The authors reported that adapted response patterns were somewhat similar (spatially correlated) to the response patterns of the original movement direction, but were also similar to the patterns of the shifted visual target. The authors suggested that M1 may, therefore, contain a mixture of motor and visual neural populations, which remain faithful to the original movement direction or shift to the altered visual target location, respectively.

In the current study, we recorded movement kinematics and fMRI activity while subjects performed "out-and-back" reaching movements to four peripheral targets. Pattern classification techniques were used to decode movement direction from the fMRI response patterns in multiple motor and visual areas. Decoding was performed before (baseline) and after applying a $45^{\circ}$ visuomotor rotation (rotated). Dissociation between visual and motor 
A

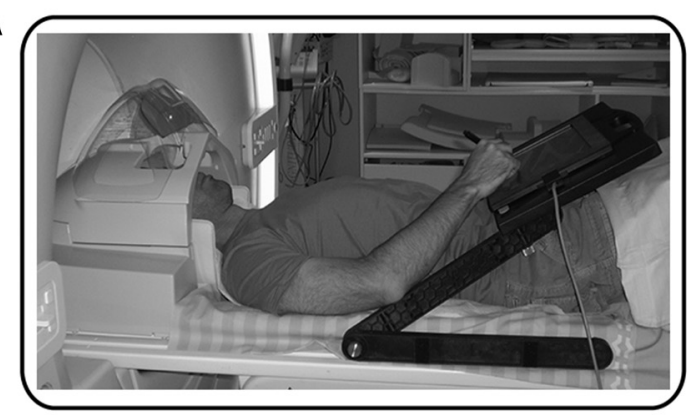

C

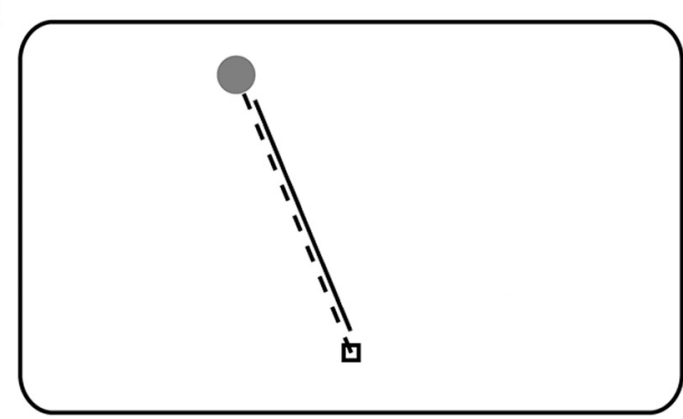

B

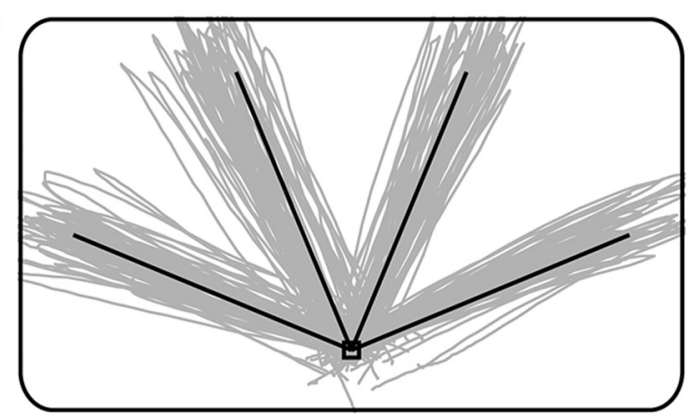

D

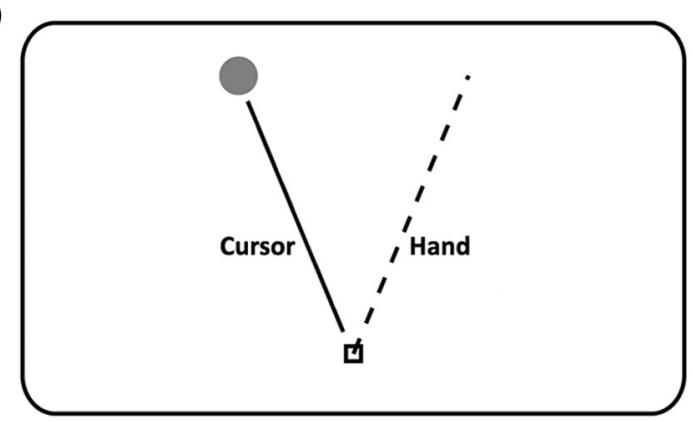

Figure 1. Experimental setup and design. $\boldsymbol{A}$, Subjects viewed a back-projected screen through a tilted mirror and performed hand movements using a stylus on an MRI-compatible digitizing tablet. $\boldsymbol{B}$, Example of movement paths from baseline trials of a typical subject. $\boldsymbol{C}$, During baseline and washout trials, hand motion and cursor motion were congruent. $\boldsymbol{D}$, During rotated trials, subjects moved their hand $45^{\circ}$ clockwise to the direction of the target to get the cursor to the target.

directional selectivity was assessed by decoding movement direction using brain activity from the rotated condition after training the classifier with trials from the baseline condition. We found that visual brain areas exhibited the same visual directional selectivity and motor areas exhibited the same motor selectivity before and after adapting to the visuomotor rotation. Most importantly, responses in medial intraparietal sulcus (mIPS) within the PPC, an intermediate visuomotor area, were altered by the visuomotor rotation and changed their directional selectivity such that it differed from the selectivity found in the baseline trials. We, therefore, suggest that neural populations in IIPS play a central role in adapting to new visual environments by encoding the new visuomotor relationship (i.e., directional transformation) imposed by the visuomotor adaption protocol.

\section{Materials and Methods}

Subjects. Ten right-handed volunteers with normal or corrected-tonormal visual acuity (5 women and 5 men; age range, 24-28 years) participated in the present experiments. The Soroka Medical Center Internal Review Board approved the experimental procedure, and written informed consent was obtained from each subject.

Experimental setup and design. Subjects lay in the scanner bore and viewed a back-projected screen through an angled mirror, which prevented any visual feedback of their hand. An MRI-compatible digitizing tablet (Hybridmojo LLC) was placed over the subject's waist and was used to track their hand movements (Fig. 1A). Subjects performed outand-back reaching movements to four targets spaced $45^{\circ}$ apart (Fig. $1 B$ ). Each trial started with the following three simultaneous events: the central target changed from red to green; the cursor appeared in the center of the screen; and one peripheral target appeared. The subject then had $2 \mathrm{~s}$ to perform the out-and-back movement, by moving the stylus pen on the tablet, after which the center target turned red, and the cursor and peripheral target disappeared. The curser gave on-line feedback of the movement at a frequency of $200 \mathrm{~Hz}$. During the intertrial interval (ITI), the center target remained, and subjects were asked to fixate and avoid any movements. There was no post-trial visual feedback or knowledge of results.
Experimental paradigm. The experiment included the following three conditions: (1) baseline, in which cursor and hand movements were aligned (Fig. 1C); (2) rotated, in which the cursor movement was rotated by $45^{\circ}$ counterclockwise (CCW) with respect to the hand movement (Fig. 1D); and (3) washout, in which the cursor and hand movements again were aligned. For each condition, the subjects completed one learning block followed by three (for baseline) or two (for rotation and washout) experimental blocks. The learning blocks were composed of trials with short ITIs (i.e., a movement was performed every $4 \mathrm{~s}$ ), and experimental blocks were composed of trials with long ITIs of $10 \mathrm{~s}$ to allow relaxation of the hemodynamic response between trials. Learning blocks were not scanned, but hand kinematics was recorded. The first learning block was used to train the subjects on the task inside the MRI bore. The other two learning blocks were used to allow the subjects to learn and unlearn the visuomotor rotation. Each of the baseline and washout blocks contained 48 trials, with 12 movements to each of the four targets. Each rotated block contained 48 trials, but with 16 movements to each of the three left targets (i.e., the right-most target was omitted). The learning block contained 60 trials with 20 or 15 movements to each target for the rotation and washout conditions, respectively.

Movement recording and analysis. Kinematic data were recorded at 200 $\mathrm{Hz}$. Trials where the error in the movement angle at peak velocity or at the end point exceeded $20^{\circ}$ or trials where movement length was $<50 \%$ or $>200 \%$ of the target location were discarded from further analysis. On average, $\sim 3 \%$ (SD, $3 \%$ ) of the trials from the baseline condition, $7 \%$ (SD, $5 \%$ ) of the trials from the rotated condition, and $5 \%$ (SD, $5 \%$ ) of the trials from the washout condition were discarded.

MRI acquisition and preprocessing. Imaging was performed using a Philips Ingenia 3 T MRI scanner located at the Soroka Medical Center in Beer-Sheva, Israel. The scanner was equipped with a 32-channel head coil, which was used for RF (radio frequency) transmission and reception. Blood oxygenation level-dependent contrast was obtained using a T2*-sensitive echoplanar imaging pulse sequence $[\mathrm{TR}=2000$ $\mathrm{ms} ; \mathrm{TE}=35 \mathrm{~ms}$; flip angle $(\mathrm{FA})=90^{\circ} ; 28$ slices; with functional voxel size of $2.6 \times 2.6 \times 3 \mathrm{~mm}$; with a $0.6 \mathrm{~mm}$ gap]. Anatomical volumes were acquired with a T1-weighted sagittal sequence image of the whole brain $\left(\mathrm{TR}=8.165 \mathrm{~ms} ; \mathrm{TE}=3.74 \mathrm{~ms} ; \mathrm{FA}=8^{\circ}\right.$; with anatomical voxel size of $1 \times 1 \times 1 \mathrm{~mm}$ ). 

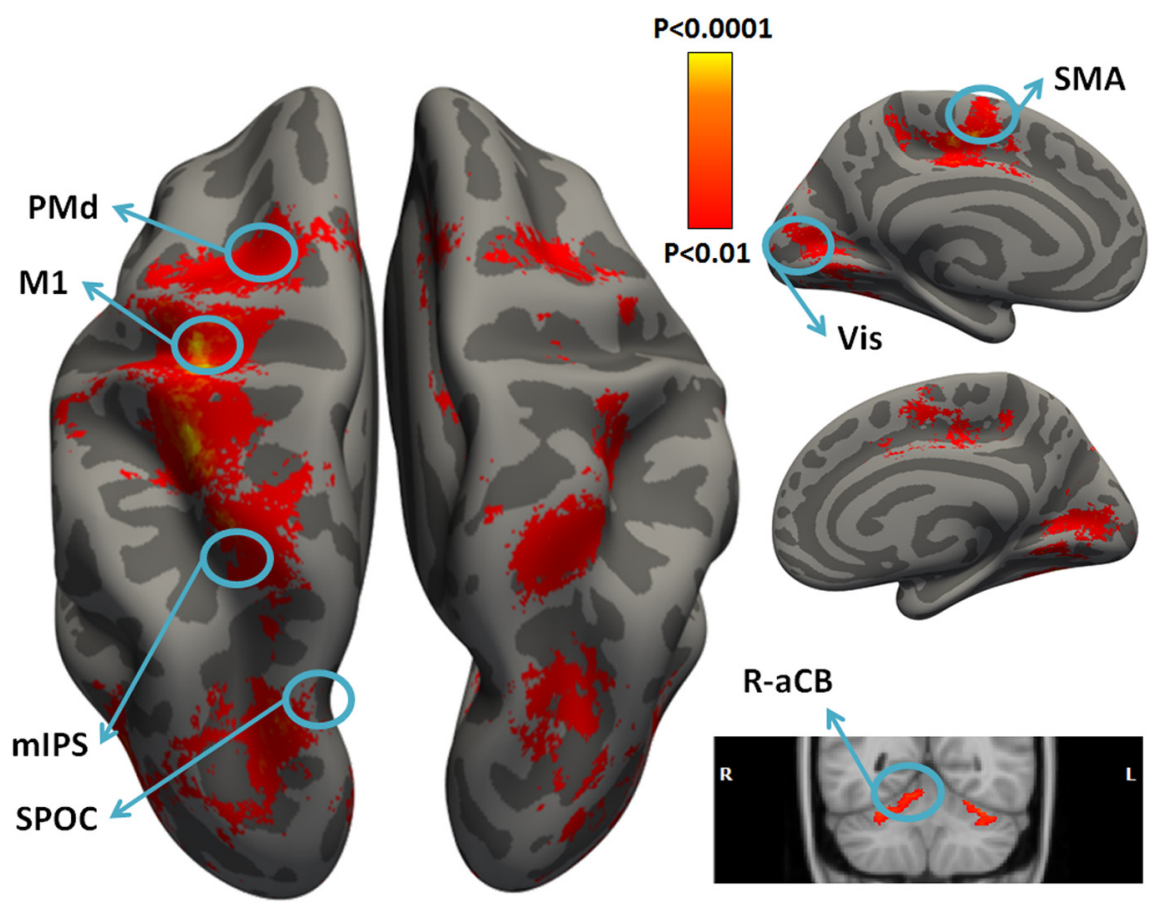

Figure 2. Regions of interest. Cortical and cerebellar areas that exhibited larger responses during movement than rest are shown in red/orange. Results calculated across all subjects (random-effects GLM) and displayed on inflated hemispheres of a template brain. The general locations of the selected ROIs are outlined in circles (actual ROIs were anatomically and functionally defined in each subject). R0Is: left M1, left PMd, left SMA, left mIPS, left SPOC, left Vis, and right aCB (R-aCB). L, Left; R, right.

MRI data were preprocessed with the Brain Voyager software package (Brain Innovation Inc.). Functional scans were subjected to slice scantime correction, 3D motion correction, and temporal high-pass filtering with a cutoff frequency of two cycles per scan. Functional images were aligned with the high-resolution anatomical volume using trilinear interpolation. No additional spatial smoothing was performed. Preprocessed data were imported to MATLAB (R2012a, MathWorks) using NeuroElf [version $0.9 \mathrm{c}$ (http://neuroelf.net)]. All further analysis was performed using custom software written in MATLAB.

Identification of regions of interest. Visual and motor brain areas were defined a priori according to a combination of anatomical and functional criteria. We analyzed six cortical regions of interest (ROIs) in the contralateral hemisphere (left hemisphere), which were individually defined in each subject's native space. ROIs included early visual cortex (Vis; located in the occipital pole), superior parieto-occipital cortex (SPOC; superior end of the parieto-occipital sulcus), mIPS (half-way up the length of the IPS), M1 (hand knob in the left central sulcus), PMd (junction of the precentral sulcus and superior frontal sulcus), and SMA (medial walls anterior to the precentral gyrus).

ROIs were constrained anatomically using the Freesurfer anatomical parcellation algorithm (http://surfer.nmr.mgh.harvard.edu; Fischl, 2012). Briefly, this process includes the removal of nonbrain tissue, and the segmentation of subcortical matter, gray matter, and white matter based on image intensity. Individual brains were registered to a spherical atlas that used individual cortical folding patterns to match brain geometry across subjects. Each brain was then parcellated into 148 cortical ROIs using the anatomical atlas of Destrieux et al. (2010). A functional analysis using a standard general linear model (GLM) was then performed to identify the voxel with the strongest response during movement execution (regardless of movement direction) in each of the relevant ROIs. The final ROI was determined using an algorithm that selected 200 functional voxels that maximized a cost function weighing the strength of movement execution response and distance from ROI center, but fulfilling the constraint that the voxels be continuous (Fig. 2).

An additional ROI was defined in the ipsilateral (right) anterior lobe of the cerebellum (aCB; Fortier et al., 1989) according to the SUIT template (http://www.icn.ucl.ac.uk/motorcontrol/imaging/propatlas.htm;
Diedrichsen et al., 2009), which was transformed into each subject's native space using FSL (http://fsl.fmrib.ox.ac.uk/fsl/fslwiki/). A functional ROI within the aCB was defined in each subject, as described above. Anatomical landmarks of all ROIs are listed in Table 1.

$M V P A$. We first estimated the response amplitude of each voxel in each trial using a GLM analysis where the GLM contained a row for every time point and a column for every trial. Each column contained a delta function at trial onset, which was convolved with a canonical hemodynamic response function. The response amplitude associated with each trial (i.e., $\beta$ value) was estimated using multiple regression, and the statistical significance of the response amplitude was estimated by computing its $t$ statistic. Voxel-by-voxel $t$ values of each trial formed a multidimensional vector with the number of dimensions equal to the number of voxels in the ROI. $t$ value rather than $\beta$-value vectors were used in all classification analyses to suppress the contribution of voxels with large trial-by-trial variability (Misaki et al., 2010). To avoid technical jargon, we subsequently use the term voxel-by-voxel fMRI response pattern to mean the $t$ value vectors.

We performed the classification analyses using both a multiclass linear discriminant analysis (LDA) classifier implemented in the statistics toolbox of MATLAB and a multiclass linear support vector machine (SVM) classifier implemented in LIBSVM (Chang and Lin, 2011). Comparison of the two classifiers was used to assess the reliability and stability of the classification results.

We trained each of the classifiers to identify the movement direction of each trial according to the voxel-by-voxel fMRI patterns in each ROI. In all cases, classifiers were trained only with trials where the movement angle error at peak velocity and end point was $<10^{\circ}$, and the movement length was $70-150 \%$ of the target distance. This ensured that classifier training was performed with trials containing the most precise movements in terms of direction and extent.

We first performed this analysis "within condition" using a "leaveone-out" validation scheme. This included training the classifier using all but one of the accurate trials, and then assessing the accuracy of the classifier by decoding the movement direction of the remaining trial. We repeated this process while leaving out each of the trials and then estimated the overall decoding accuracy by computing the proportion of accurately decoded trials for each condition (i.e., baseline, rotated, and washout). We then performed a "between-condition" analysis where we trained the classifier on all of the accurate baseline trials and decoded the movement direction in all trials from the rotated or washout conditions (i.e., trials where the movement angle error at peak velocity and end point was $<20^{\circ}$, and the movement length was $50-200 \%$ of the target distance). This enabled us to demonstrate that accurate decoding across conditions was possible even with more relaxed inclusion criteria. Decoding accuracy was estimated as the proportion of trials that were accurately decoded.

The number of trials used to train each of the classifiers was not necessarily balanced across targets (there were differences of up to six trials across targets). To prevent classification bias toward over-represented targets, the classifier was set with a uniform probability prior. We confirmed this methodological step and the consequential results by repeating the analysis on a fully balanced subset of trials, which yielded equivalent results when using either the LDA or SVM classifiers.

To assess the statistical significance of decoding accuracy in both within- and between-condition analyses, we performed a randomization test that was identical to the classification analysis described above except 
Table 1. Mean Rol Talairach coordinates

\begin{tabular}{lllr}
\hline & \multicolumn{2}{l}{ Talairach coordinates } & \multicolumn{1}{c}{} \\
\cline { 2 - 4 } R0I name & $x$ & $y$ & -6.1 \\
\hline L Vis & -20.9 & -88.5 & 36.3 \\
L SPOC & -16.2 & -78.2 & 43.3 \\
L mIPS & -30 & -55.4 & 50.2 \\
L M1 & -31.9 & -25.4 & 52.9 \\
L PMd & -26.3 & -10.4 & 60 \\
L SMA & -4.7 & -11.9 & -13.8 \\
RaCB & 11.8 & -47.9 &
\end{tabular}

L, Left; R, right.

that we randomly shuffled the movement labels before training the classifier. We ran this analysis 2000 times for each subject separately, reshuffling the movement labels each time, and then computed the mean across subjects for each iteration. The mean decoding accuracy across subjects was considered significantly larger than chance if it exceeded the 97.5th percentile of the null/chance distribution for each ROI. Accordingly, all statistical tests used in all graphs and all analyses are based on the permutation tests and not on theoretical chance levels.

We used false discovery rate (FDR) correction (Benjamini and Hechtlinger, 2014) to control for the multiple-comparisons problem both in the ROI analysis (across ROIs in each condition) and in the searchlight analysis (across voxels in each condition).

Directional tuning curves. This analysis examined the decoding outcome of single trials as a function of their angle from the actual target. The possible angular outcomes for the four targets ranged from $0^{\circ}$ to $\pm 135^{\circ}$. For example, a movement to the left-most target could be decoded as $0^{\circ}$ (accurate), $45^{\circ}, 90^{\circ}$, or $135^{\circ}$, while a movement to the rightmost target could be decoded as $0^{\circ},-45^{\circ},-90^{\circ}$, or $-135^{\circ}$. There were, therefore, seven possible angular outcomes, each with a different chance level according to the availability of the angles across targets. The presented tuning curves for each ROI (see Fig. 7) show the decoding outcomes for each angle relative to its chance level.

Searchlight analysis. In addition to the ROI analysis, we used a searchlight analysis (Kriegeskorte et al., 2006) to map classifier decoding accuracies across the entire brain. Clusters of 27 functional voxels were defined by creating a volumetric cube with an edge length of 3 voxels around each gray matter voxel. An LDA classification analysis was performed for each cluster, as described above, such that each gray matter voxel was associated with a decoding accuracy value yielding a decoding accuracy map. The searchlight analysis was performed in the native space of each subject. Decoding accuracy maps of all subjects were transformed to a standard cortical surface using Freesurfer, and a $t$ test was used to determine whether each vertex exhibited significant above-chance decoding accuracies across subjects. In the cerebellar searchlight analysis, the searchlight decoding accuracy maps of all subjects were transformed using the Talairach transformation and a $t$ test was used to assess statistical significance in each voxel of the cerebellar cortex.

\section{Results}

\section{Visuomotor adaptation}

In the rotated condition, the movement of the cursor was rotated CCW by $45^{\circ}$, and the subjects had to learn to compensate by moving their hand in a $45^{\circ}$ clockwise direction to accurately reach the appropriate target (Fig. 1). In the washout condition, hand and cursor directions were aligned again and subjects relearned to move in the original directions. All subjects learned to accurately move the cursor to the targets such that errors were reduced rapidly at first and more slowly thereafter in both the rotated and washout conditions. The mean time course of error reduction across subjects during the learning blocks was well fit by a double exponential function $\left(r^{2}=0.81\right.$; Fig. 3$)$.

\section{Decoding movement direction}

We assessed the decoding accuracy of movement directions in each of eight visual and motor brain regions that were defined according to anatomical constraints and functional responses in each subject separately (see Materials and Methods). In the first analysis, we evaluated the decoding accuracy within each of the baseline, rotated, and washout conditions. SVM and LDA classifiers were trained to identify movement direction according to the voxel-by-voxel response patterns of single trials, and decoding accuracy was assessed using a leave-one-out validation scheme (see Materials and Methods). All results presented are for the LDA classifier, though similar results were found with the SVM classifier. Statistical significance was assessed using a randomization analysis, and the FDR correction was used to address the multiple-comparisons problem (see Materials and Methods).

The mean decoding accuracies for baseline trials were well above chance level (25\%) for all ROIs (Fig. 4). Vis showed the highest decoding accuracies $(73 \%, p<0.001)$, followed by SPOC (52\%, $p<0.001)$, M1 (42\%, $p<0.001)$, $\operatorname{mIPS}(40 \%, p<0.001)$, PMd (40\%, $p<0.001)$, SMA (39\%, $p<0.001)$, and aCB $(37 \%$, $p<0.001)$. These results showed that directional selectivity was clearly apparent in the voxel-by-voxel fMRI patterns of multiple visual and motor system areas.

Equivalent within-condition classification analyses of the rotated and washout trials showed decoding accuracies of similar strengths and significance across all ROIs (Fig. 4A). Unlike the baseline and washout conditions, where movements were performed to four targets and the chance decoding accuracy was $25 \%$, in the rotated condition movements were performed to only three targets such that the chance decoding accuracy was $33 \%$. To compare values across conditions, we adjusted the accuracy rates of the rotated condition as follows: accuracy rates were divided by the three-way chance level (33\%) and multiplied by the four-way chance level (25\%). The early visual area continued to show the highest accuracies (58\% and 67\%, $p<0.001$ for adjusted rotated and washout conditions respectively), followed by SPOC ( $45 \%$ and $47 \%, p<0.001)$, mIPS $(39 \%$ and $40 \%$, $p<$ $0.001)$, M1 (42\% and 36\%, $p<0.001$ ), PMd (35\% and $37 \%, p<$ 0.001 ), SMA ( $40 \%$ and $34 \%, p<0.001$ ), and $\mathrm{CCB}(37 \%$ and $37 \%$, $p<0.001)$.

This analysis shows that decoding accuracies did not change much throughout the experiment, which lasted $>1$ h. The washout condition was performed at the end of the experiment when subjects were likely to be more tired and less cooperative, yet decoding accuracies were still significantly above chance, attesting to the robustness of the directional selectivity found in the fMRI response patterns.

\section{Decoding movement direction across conditions}

Given the robustness of the directional selectivity within each condition, we assessed the effects of visuomotor adaptation on directional selectivity across conditions. We trained a classifier to identify movement direction using the accurate baseline trials and tested its ability to decode movement direction in all of the rotated trials (see Materials and Methods). Since the angle of the visuomotor rotation was $45^{\circ} \mathrm{CCW}$, each trial in the rotated condition could have been decoded in two ways (Fig. 1D). The first was according to the visual location of the target (i.e., visual directional selectivity), and the second was according to the direction of the hand movement (i.e., motor directional selectivity). We, therefore, performed the decoding procedure twice, as follows: once according to the location of the visual target, and again according to the direction of hand movement. This analysis enabled us to dissociate ROIs with visual directional selectivity, where trials were accurately decoded according to baseline visual target location, from ROIs with motor directional selectivity, 


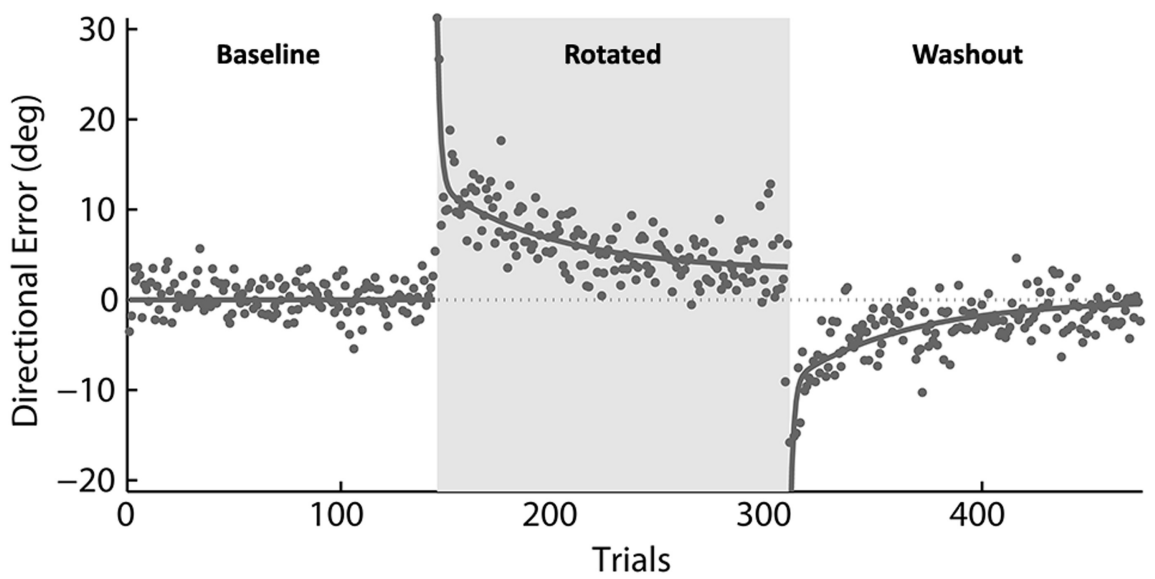

Figure 3. Mean learning curves. The mean directional error across subjects is plotted per trial and fit with a double exponential function $\left(\tau_{\mathrm{f}}=4.3\right.$ trails, $\tau_{\mathrm{s}}=33.2$ trails). Baseline, first 144 trials; rotated, trials 145-310; washout, trials 311- 476 .

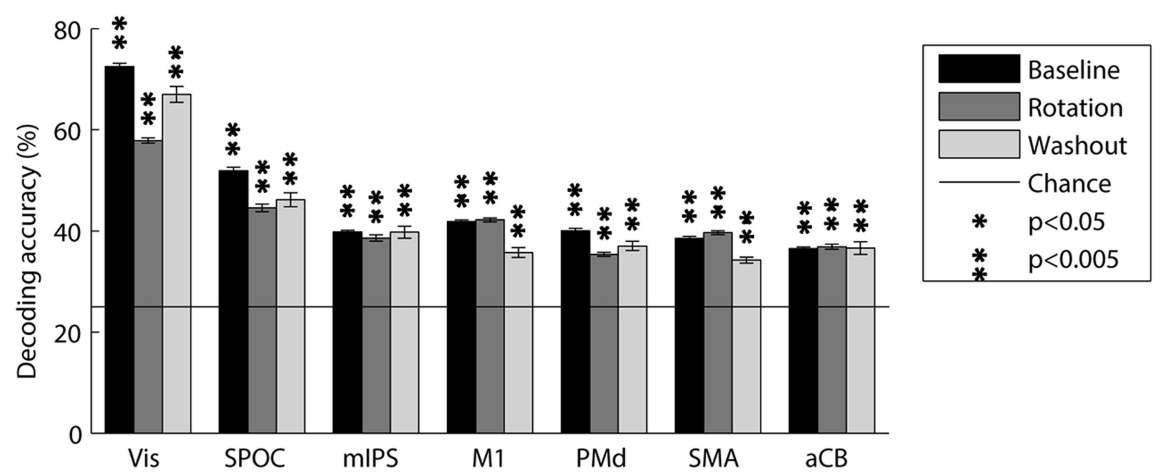

Figure 4. Mean decoding accuracies across subjects. Within condition decoding was performed for each of the baseline (black), rotated (dark gray), and washout (light gray) conditions separately using a leave-one-out validation scheme. Solid line indicates chance level (25\%, four movement directions). Error bars indicate SEM across subjects. Asterisks indicate significant above-chance decoding accuracies ( $p<0.05$, randomization test, FDR corrected for multiple comparisons).

where trials were decoded according to baseline movement direction.

Response patterns in visual brain areas were accurately decoded according to visual target location, but not according to hand movement direction, while response patterns in motor brain areas showed the opposite result (Fig. 5). Decoding accuracies according to visual target location were significantly above chance levels in Vis $(58 \%, p<0.001)$ and SPOC $(40 \%, p<$ 0.001 ), and decoding accuracies according to hand movement direction were significantly above chance levels in M1 $(37 \%, p<$ $0.001)$, PMd (30\%, $p<0.02)$, SMA (32\%, $p<0.002)$, and $\mathrm{aCB}$ (33\%, $p<0.001)$. The significance of decoding accuracies reported above was estimated using a randomization analysis, and all $p$ values were FDR corrected (see Materials and Methods).

Response patterns in mIPS during the rotated condition were not accurately decoded according to either visual or motor directions in the baseline condition (28\% and $27 \%$, respectively; $p>$ 0.2 ). Note that the mIPS area did exhibit significant withincondition decoding accuracies during the baseline, rotated, and washout conditions as well as significant between-condition decoding accuracies when using the washout trials (see below). This demonstrates that directional selectivity was apparent in the response patterns of mIPS throughout the experiment, yet this selectivity was altered following visuomotor rotation in a manner that did not match either the motor movement direction or visual target location.
This double dissociation across visual and motor ROIs was also clearly apparent in an ANOVA where there was a significant interaction $(p<0.001)$ between ROI and decoding condition (i.e., decoding rotated trials according to their visual target location vs movement direction).

To assess the robustness of these results, we also performed the opposite analysis where we used a classifier trained with rotated trials to decode the movement direction of baseline trials. This was performed once when training the classifier according to visual target location (visual classifier), and again when training the classifier according to movement direction (motor classifier). This analysis yielded equivalent findings such that response patterns in visual areas (Vis and SPOC) enabled accurate decoding of baseline trials when using the visual classifier, while response patterns in motor areas (M1, PMd, SMA, and $\mathrm{aCB}$ ) enabled accurate decoding of baseline trials when using the motor classifier. Response patterns in mIPS did not enable accurate decoding of baseline trials according to either classifier.

Finally, to demonstrate the robustness of directional selectivity over time (i.e., across early and late trials of the experiment), we also decoded washout trials using the same classifier trained on the accurate baseline trials. Mean accuracies were again well above chance for all ROIs, as follows: Vis, $67 \%(p<0.001)$; SPOC, $46 \%(p<0.001)$; mIPS, $40 \%(p<0.001)$; M1, 36\% ( $p<0.001)$; PMd, 37\% $(p<0.001)$; SMA, 34\% $(p<$ $0.001)$; and $\mathrm{aCB}, 37 \%(p<0.001)$.

\section{Directional tuning curves}

In an additional analysis, we estimated directional "tuning curves" for each of the ROIs by quantifying trial-by-trial decoding outcomes (Fig. 6). Note that the chance level for each of the seven possible angular outcomes $\left(-135^{\circ},-90^{\circ},-45^{\circ}, 0^{\circ}, 45^{\circ}\right.$, $90^{\circ}$, and $135^{\circ}$ ) differed across targets since there were only four possible outcomes per target (e.g., the right-most target could be decoded only as $-135^{\circ},-90^{\circ},-45^{\circ}$, and $0^{\circ}$ ), we therefore normalized the decoding percentage of each target according to its possible outcomes and then collapsed the results across targets. When computing the decoding outcomes of washout trials based on a classifier trained with baseline trials, all brain areas, including mIPS, exhibited decoding outcomes that were significantly tuned to the true movement/target direction of the trial (Fig. $6 \mathrm{~A}$ ). When performing the same analysis with the rotated trials (Fig. $6 B)$, however, the early visual area (i.e., the Vis) and the SPOC showed clear and significant tuning to the direction of the visual target (i.e., decoding outcomes were aligned to the target direction), while the motor areas (M1, PMd, SMA, and aCB) showed clear and significant tuning to the actual movement direction (i.e., decoding outcomes were aligned to the movement direction). In contrast, area mIPS was not significantly tuned to either target or movement directions. 


\section{Searchlight decoding}

We used a whole-brain searchlight analysis (Kriegeskorte et al., 2006) to assess directional selectivity across the entire cortical surface without restricting the analysis to a priori ROIs. We used a volumetric searchlight cube of 27 functional voxels across the cortical gray matter. For each of the searchlight cubes, we performed between-condition decoding (training the classifier on baseline trials and then decoding trials from the rotated condition), as described above in the ROI analyses. The significance of decoding accuracies in each voxel was assessed by a $t$ test across subjects and was corrected for multiple comparisons using FDR correction.

The searchlight map in the left hemisphere shows results that are complementary to those described in the ROI analysis and demonstrates the spatial selectivity of the brain areas where directional encoding was evident (Fig. 7A). Significant decoding according to movement direction was evident in left M1, PMd, and SMA, while significant decoding according to target direction was evident in left visual cortex. Note that between-condition decoding accuracies were not apparent anywhere in the entire IPS, demonstrating that directional selectivity was indeed altered throughout parietal cortex after visuomotor adaptation. When applying the searchlight analysis to the washout condition (Fig. 7B), parietal areas do exhibit significant decoding accuracies, demonstrating that directional selectivity was maintained in parietal cortex throughout the experiment yet was altered following visuomotor adaptation. These results validate the ROI results using far smaller clusters of voxels for the classification and decoding procedures.

Note that the searchlight map reveals similarly significant decoding accuracies in the ipsilateral right hemisphere, which suggests that directional selectivity is apparent in similar brain areas across both hemispheres. This may not have been expected given the assumption that the motor system is strongly lateralized.

Performing the searchlight analysis in the cerebellum, we found significant decoding accuracies for movement direction only in right lobule $\mathrm{V}$ (which overlaps with the aCB ROI) as well as in right Crus I and Crus II (Fig. 7A). The cerebellar analysis also revealed significant decoding accuracies in the contralateral cerebellar hemisphere, attesting to the potential bilateral nature of directional selectivity.

\section{Discussion}

How does the human brain adapt to altered visual surroundings? The common view is that our brain maintains a visuomotor map that transforms visual locations in extrinsic coordinates to motor commands in intrinsic coordinates (Shabbott and Sainburg, 2010). When the visual environment changes, such visuomotor maps need to be updated to maintain accuracy. In the current study, we examined what happens to directional tuning/selectivity following a $45^{\circ}$ visuomotor rotation, where the movement direction was dissociated from the visual target location. While directionally selective response patterns in early visual areas and SPOC remained faithful to the visual target location, regardless of movement direction, directionally selective response patterns in M1, PMd, SMA, and anterior cerebellum remained faithful to movement direction, regardless of visual target location (Figs. 4, 5). Most importantly, directionally selective response patterns in mIPS did not remain faithful to either visual location or movement direction. Instead, directionally selective mIPS response patterns in the rotated condition were altered by the learned visuomotor transformation and only returned to their original directional tuning once the visuomotor adaptation had been washed out (Figs. 5, 7). We, therefore, suggest that sustained (learned) changes in visuomotor mapping following visuomotor adaptation are stored by neural populations located in PPC. Similar role has been hypothesized previously based on theoretical considerations (Tanaka et al., 2009).

\section{Directional selectivity changes in parietal cortex}

When interpreting the change in directional selectivity of the fMRI response patterns in mIPS following adaptation learning, it is important to consider what may cause the fMRI response patterns to change. Our assumption is that fMRI response patterns are generated by the responses of distributed neural populations 


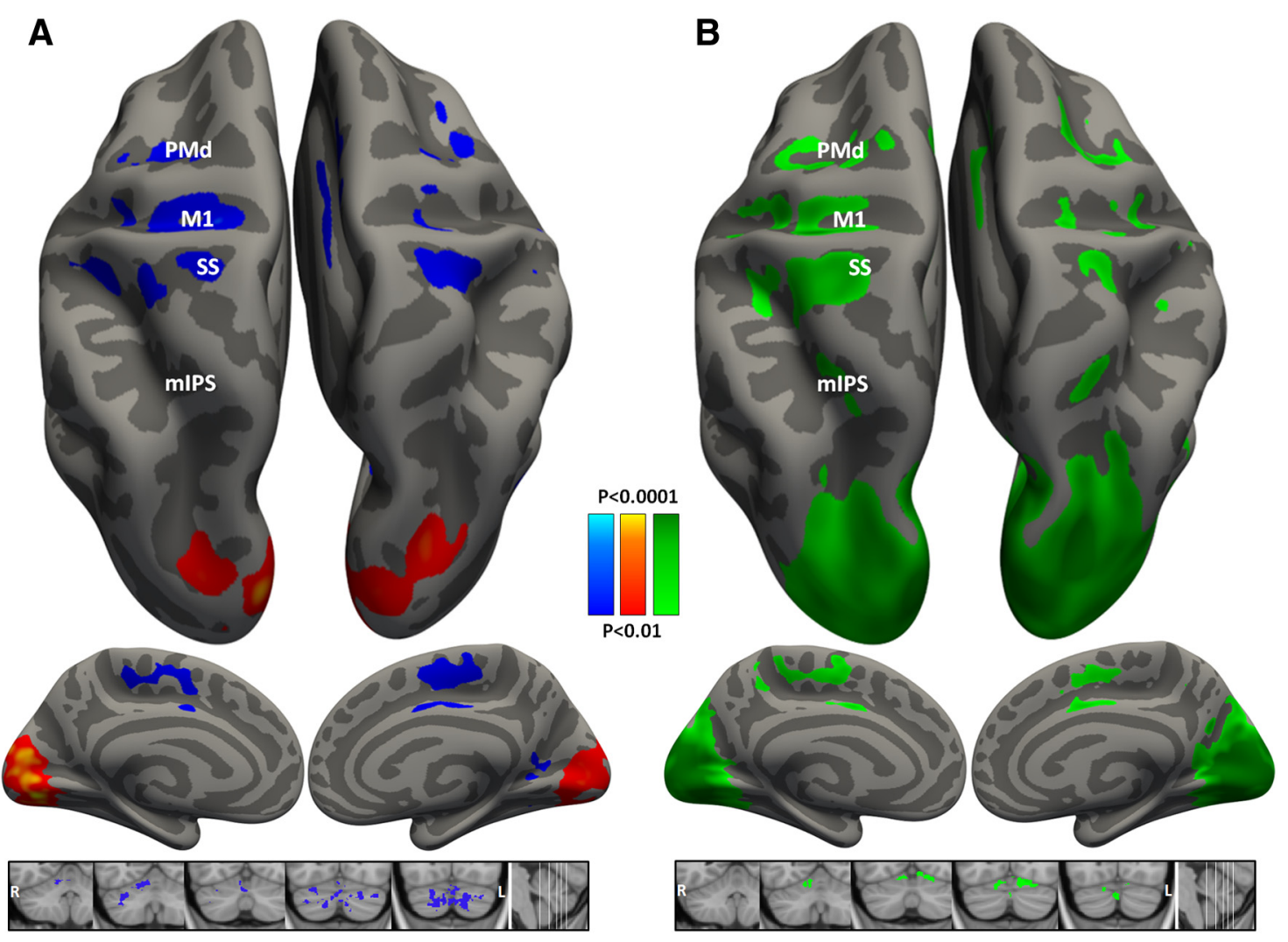

Figure 7. Whole-brain searchlight analyses. Cortical vertices and cerebellar voxels with decoding accuracies that were significantly above chance $(>25 \%)$ across subjects are marked on inflated hemispheres of a template brain. For each cluster of vertices (cortical gray matter) or voxels (cerebellum), the classifier was trained on baseline trials. $A, B$, Trials from the rotated condition were decoded according to their motor movement direction (blue, $p<0.01 \mathrm{FDR}$ corrected) or their visual target location (red, $p<0.01 \mathrm{FDR}$ corrected; $A$ ); and trials from the washout condition (green, $p<0.01$ FDR corrected; $\boldsymbol{B})$.

that are located within the examined voxels. A change in the fMRI pattern would, therefore, imply a change in the identity of the responding neural population. If one assumes that mIPS contains a mixture of interconnected directionally selective visuomotor neurons, the changes in fMRI pattern between the baseline and rotated condition are likely to reflect changes in the neural populations that respond to each direction. Such a change may happen when visuomotor neurons change their directional tuning and/or when the synaptic weighting between visual and motor populations changes (Tanaka et al., 2009). Final proof would require recording single-neuron responses before and after adaptation, and assessing the changes in their tuning curves. Such an experiment has not been performed so far and is highly warranted.

The SPOC is a more posterior parietal region, which is thought to be involved in the preparation and execution of visually guided reaching movements (Galletti et al., 1997; Prado et al., 2005; Cavina-Pratesi et al., 2010; Gallivan et al., 2011). In agreement with a previous fMRI study (Fernandez-Ruiz et al., 2007), our results revealed that the directionally selective response patterns in this area were not altered by the visuomotor adaptation and continued to enable accurate decoding of target location rather than movement direction following adaptation learning.

\section{Directional selectivity in motor cortices}

Response patterns in M1, PMd, and SMA exhibited equivalent directional selectivity according to movement direction before and after the visuomotor adaptation, regardless of the location of the target. These results suggest that the responses of neural pop- ulations in these areas remain faithful to the original movement direction and are not altered when individuals learn a new visuomotor mapping. While a previous fMRI study (Eisenberg et al., 2011) did report that M1 response patterns exhibit both motor and visual directional selectivity, most electrophysiology studies in primates have reported that, when executing movements after adaptation learning, the majority of M1 (Shen and Alexander, 1997) and SMA (Crutcher et al., 2004) neurons retain their original motor directional tuning. An important difference between our study and the previous one is that in the previous study subjects moved a joystick and performed movements only with the wrist, while our subjects made naturalistic out-and-back reaching movements that involved movements of the entire arm, which may have generated larger and more robust motor neural responses. Note that the use of full arm movements is the common experimental choice in the visuomotor adaptation literature (Krakauer et al., 1999; Ghilardi et al., 2000; Neva and Henriques, 2013; Taylor and Ivry, 2013).

\section{Directional selectivity in the cerebellum}

As in the motor system areas described above, fMRI response patterns in the cerebellum enabled accurate decoding of movement direction regardless of target location. This was evident in two areas within the cerebellum: right lobule V (Figs. 4, 5, 7); and right Crus I and Crus II (Fig. 7). These results suggest that the neural population within the cerebellum faithfully encodes movement direction in a similar manner before and after learning the visuomotor rotation (Fig. 5). While the cerebellum is known to play a critical role in learning visuomotor adaptation, 
as demonstrated by humans with cerebellar lesions in lobule $\mathrm{V}$ or Crus I (Werner et al., 2010; Donchin et al., 2012), our results suggest that sustained changes associated with the learned visuomotor mapping are stored in the MIPS and not in the cerebellum.

\section{Different types of visuomotor learning}

Current theories of visuomotor adaptation suggest that different aspects of adaptation may be learned in different ways and potentially by using different underlying neural mechanisms. For example, it has been proposed that adaptation includes a fast process that learns and forgets quickly, and a slow process that learns slowly and retains (Smith et al., 2006). In addition, adaptation can be learned by a sudden large visuomotor dissociation or by introducing gradual increments in the visuomotor dissociation (Kagerer et al., 1997). In the current study, we used a sudden rotation task. Future assessments of directional selectivity changes following a gradual rotation task will be able to determine whether sustained directional selectivity changes are apparent in MIPS and/or in other brain areas, thereby elucidating the neural mechanisms involved in the learning outcome of each adaptation paradigm. With respect to the distinction between fast and slow learning processes (Smith et al., 2006), our study examined brain activity only after adaptation learning had been completed and behavioral accuracy had reached an asymptote. By that time, the fast process had presumably already decayed and brain activity potentially reflects mostly the slow learning process and sustained changes associated with completed learning. While fast learning processes may indeed be cerebellar dependent (Smith et al., 2006), our results seem to suggest that slow learning process and sustained adaptation outcomes are apparent in PPC rather than cerebellar responses. This is in line with a recent study (Galea et al., 2011) using transcranial direct current stimulation (tDCS), which found that, while cerebellar tDCS caused faster adaptation to visuomotor transformation, cortical tDCS (applied over M1) increased the retention.

\section{Bilateral decoding}

While the motor system is thought to be strongly lateralized, neural populations in M1 and SMA are known to exhibit bilateral directional selectivity (Donchin et al., 1998). This finding was recently replicated in humans using fMRI (Fabbri et al., 2010) and is also demonstrated in our results (Fig. 7). Moreover, the searchlight analysis revealed that bilateral directional selectivity is apparent not only in M1, but also in PMd, mIPS, SPOC, and somatosensory cortices. We speculate that bilateral directional selectivity may be the product of strong interhemispheric connectivity or of independent movement-related activity in both hemispheres.

\section{Limitations of the study}

The current study is limited in two important manners. First, since response patterns were assessed following adaptation learning and not during adaptation learning, we can only assess the outcome of learning rather than actual learning dynamics. Given the quick nature of sudden adaptation learning, the sluggish nature of the measured hemodynamic responses, and the necessity for multiple trials when performing statistical analyses, studying adaptation learning using fMRI is a particularly large challenge, which may be addressed to some extent through the use of a gradual adaptation learning protocol.

A second important limitation of the current study is that we did not control for eye movements. Multiple fMRI studies have reported that parietal areas including SPOC and MIPS are in- volved in planning and executing both reaching movements and saccades (Medendorp et al., 2003; Levy et al., 2007; Beurze et al., 2009; Filimon et al., 2009; Vesia and Crawford, 2012; Leoné et al., 2014). Since we did not control for saccades, there is a chance that saccade-related activity may have affected our ability to decode movement/target direction in different brain areas. Future studies may address this issue by enforcing a strict fixation policy or by eliminating visual feedback from some of the trials.

\section{Conclusions}

Understanding how the human motor system adjusts flexibly to the numerous ever-changing variables in our environment is a fundamental question in motor control. The results of the current study advance our understanding of this topic by demonstrating that adaptation learning leads to a change in the directionally selective responses of mIPS. While motor and visual brain areas retain faithful directional representations of the movement direction and target location, respectively, responses in MIPS seem to represent the new visuomotor mapping that had been learned during adaptation.

\section{References}

Benjamini Y, Hechtlinger Y (2014) Discussion: an estimate of the sciencewise false discovery rate and applications to top medical journals by Jager and Leek. Biostatistics 15:13-16. CrossRef Medline

Beurze SM, de Lange FP, Toni I, Medendorp WP (2009) Spatial and effector processing in the human parietofrontal network for reaches and saccades. J Neurophysiol 101:3053-3062. CrossRef Medline

Cavina-Pratesi C, Monaco S, Fattori P, Galletti C, McAdam TD, Quinlan DJ, Goodale MA, Culham JC (2010) Functional magnetic resonance imaging reveals the neural substrates of arm transport and grip formation in reach-to-grasp actions in humans. J Neurosci 30:10306-10323. CrossRef Medline

Chang C-C, Lin C-J (2011) LIBSVM: a library for support vector machines. ACM Trans Intell Syst Technol 6(27). CrossRef

Cowper-Smith C, Lau E, Helmick C, Eskes G, Westwood D (2010) Neural coding of movement direction in the healthy human brain. PLoS One 5:313330. CrossRef Medline

Crutcher MD, Russo GS, Ye S, Backus DA (2004) Target-, limb-, and context-dependent neural activity in the cingulate and supplementary motor areas of the monkey. Exp Brain Res 158:278-288. CrossRef

Destrieux C, Fischl B, Dale A, Halgren E (2010) Automatic parcellation of human cortical gyri and sulci using standard anatomical nomenclature. Neuroimage 53:1-15. CrossRef Medline

Diedrichsen J, Balsters JH, Flavell J, Cussans E, Ramnani N (2009) A probabilistic MR atlas of the human cerebellum. Neuroimage 46:39-46. CrossRef Medline

Dinstein I, Gardner JL, Jazayeri M, Heeger DJ (2008) Executed and observed movements have different distributed representations in human aIPS. J Neurosci 28:11231-11239. CrossRef Medline

Donchin O, Gribova A, Steinberg O, Bergman H, Vaadia E (1998) Primary motor cortex is involved in bimanual coordination. Nature 395:274-278. CrossRef Medline

Donchin O, Rabe K, Diedrichsen J, Lally N, Schoch B, Gizewski ER, Timmann D (2012) Cerebellar regions involved in adaptation to force field and visuomotor perturbation. J Neurophysiol 107:134-147. CrossRef Medline

Eisenberg M, Shmuelof L, Vaadia E, Zohary E (2010) Functional organization of human motor cortex: directional selectivity for movement. J Neurosci 30:8897-8905. CrossRef Medline

Eisenberg M, Shmuelof L, Vaadia E, Zohary E (2011) The representation of visual and motor aspects of reaching movements in the human motor cortex. J Neurosci 31:12377-12384. CrossRef Medline

Fabbri S, Caramazza A, Lingnau A (2010) Tuning curves for movement direction in the human visuomotor system. J Neurosci 30:13488-13498. CrossRef Medline

Fernandez-Ruiz J, Goltz HC, DeSouza JF, Vilis T, Crawford JD (2007) Human parietal "reach region" primarily encodes intrinsic visual direction, not extrinsic movement direction, in a visual-motor dissociation task. Cereb Cortex 17:2283-2292. CrossRef Medline 
Filimon F, Nelson JD, Huang RS, Sereno MI (2009) Multiple parietal reach regions in humans: cortical representations for visual and proprioceptive feedback during on-line reaching. J Neurosci 29:2961-2971. CrossRef Medline

Fischl B (2012) FreeSurfer. Neuroimage 62:774-781. CrossRef Medline

Fortier PA, Kalaska JF, Smith AM (1989) Cerebellar neuronal activity related to whole-arm reaching movements in the monkey. J Neurophysiol 62:198-211. Medline

Fu QG, Suarez JI, Ebner TJ (1993) Neuronal specification of direction and distance during reaching movements in the superior precentral premotor area and primary motor cortex of monkeys. J Neurophysiol 70:20972116. Medline

Galea JM, Vazquez A, Pasricha N, de Xivry JJ, Celnik P (2011) Dissociating the roles of the cerebellum and motor cortex during adaptive learning: the motor cortex retains what the cerebellum learns. Cereb Cortex 21:17611770. CrossRef Medline

Galletti C, Fattori P, Kutz DF, Battaglini PP (1997) Arm movement-related neurons in the visual area V6A of the macaque superior parietal lobule. Eur J Neurosci 9:410-413. CrossRef Medline

Gallivan JP, McLean DA, Smith FW, Culham JC (2011) Decoding effectordependent and effector-independent movement intentions from human parieto-frontal brain activity. J Neurosci 31:17149-17168. CrossRef Medline

Georgopoulos AP, Kalaska JF, Caminiti R, Massey JT (1982) On the relations between the direction of two-dimensional arm movements and cell discharge in primate motor cortex. J Neurosci 2:1527-1537. Medline

Ghilardi M, Ghez C, Dhawan V, Moeller J, Mentis M, Nakamura T, Antonini A, Eidelberg D (2000) Patterns of regional brain activation associated with different forms of motor learning. Brain Res 871:127-145. CrossRef Medline

Kadmon Harpaz N, Flash T, Dinstein I (2014) Scale-invariant movement encoding in the human motor system. Neuron 81:452-462. CrossRef Medline

Kagerer FA, Contreras-Vidal JL, Stelmach GE (1997) Adaptation to gradual as compared with sudden visuo-motor distortions. Exp Brain Res 115: 557-561. CrossRef Medline

Kalaska JF, Caminiti R, Georgopoulos AP (1983) Cortical mechanisms related to the direction of two-dimensional arm movements: relations in parietal area 5 and comparison with motor cortex. Exp Brain Res 51:247260. Medline

Krakauer JW, Ghilardi MF, Ghez C (1999) Independent learning of internal models for kinematic and dynamic control of reaching. Nat Neurosci 2:1026-1031. CrossRef Medline

Kriegeskorte N, Goebel R, Bandettini P (2006) Information-based functional brain mapping. Proc Natl Acad Sci U S A 103:3863-3868. CrossRef Medline

Lee D, Quessy S (2003) Activity in the supplementary motor area related to learning and performance during a sequential visuomotor task. J Neurophysiol 89:1039-1056. CrossRef Medline

Leoné FT, Heed T, Toni I, Medendorp WP (2014) Understanding effector selectivity in human posterior parietal cortex by combining information patterns and activation measures. J Neurosci 34:7102-7112. CrossRef Medline

Levy I, Schluppeck D, Heeger DJ, Glimcher PW (2007) Specificity of human cortical areas for reaches and saccades. J Neurosci 27:4687-4696. CrossRef Medline

Medendorp WP, Goltz HC, Vilis T, Crawford JD (2003) Gaze-centered updating of visual space in human parietal cortex. J Neurosci 23:6209-6214. Medline

Misaki M, Kim Y, Bandettini PA, Kriegeskorte N (2010) Comparison of multivariate classifiers and response normalizations for patterninformation fMRI. Neuroimage 53:103-118. CrossRef Medline

Neva JL, Henriques DY (2013) Visuomotor adaptation and generalization with repeated and varied training. Exp Brain Res 226:363-372. CrossRef Medline

Prado J, Clavagnier S, Otzenberger H, Scheiber C, Kennedy H, Perenin MT (2005) Two cortical systems for reaching in central and peripheral vision. Neuron 48:849-858. CrossRef Medline

Shabbott BA, Sainburg RL (2010) Learning a visuomotor rotation: simultaneous visual and proprioceptive information is crucial for visuomotor remapping. Exp Brain Res 203:75-87. CrossRef Medline

Shen L, Alexander GE (1997) Neural correlates of a spatial sensory-tomotor transformation in primary motor cortex. J Neurophysiol 77:11711194. Medline

Smith MA, Ghazizadeh A, Shadmehr R (2006) Interacting adaptive processes with different timescales underlie short-term motor learning. PLoS Biol 4:e179. CrossRef Medline

Tanaka H, Sejnowski TJ, Krakauer JW (2009) Adaptation to visuomotor rotation through interaction between posterior parietal and motor cortical areas. J Neurophysiol 102:2921-2932. CrossRef Medline

Taylor JA, Ivry RB (2013) Context-dependent generalization. Front Hum Neurosci 7:171. CrossRef Medline

Toxopeus CM, de Jong BM, Valsan G, Conway BA, Leenders KL, Maurits NM (2011) Direction of movement is encoded in the human primary motor cortex. PLoS One 6:e27838. CrossRef Medline

Vesia M, Crawford JD (2012) Specialization of reach function in human posterior parietal cortex. Exp Brain Res 221:1-18. CrossRef Medline

Werner S, Bock O, Gizewski ER, Schoch B, Timmann D (2010) Visuomotor adaptive improvement and aftereffects are impaired differentially following cerebellar lesions in SCA and PICA territory. Exp Brain Res 201:429439. CrossRef Medline

Wiestler T, Waters-Metenier S, Diedrichsen J (2014) Effector-independent motor sequence representations exist in extrinsic and intrinsic reference frames. J Neurosci 34:5054-5064. CrossRef Medline 